\title{
Stimulus redundancy and immediate recall
}

\author{
JOHN C. JAHNKE, RONALD H. NOW ACZYK, and WILLIAM WOZNIAK \\ Miami University, Oxford, Ohio 45056
}

\begin{abstract}
This study examined immediate recall in two stimulus prefix and two stimulus suffix conditions and in a condition that combined a prefix and suffix. Suffixes and the combination of a prefix with a suffix interfered more with recall overall than did prefixes. Performance in each of the conditions that included a prefix was significantly better overall than in appropriate control conditions, in which interference was augmented by a redundant element in recall. It was suggested that prefixes and suffixes lie operationally on a continuum and that their effects result from the subject's inability to dissociate the redundant element from the memory series. However, the location of redundancy imposes different processing requirements that differentially influence recall.
\end{abstract}

This experiment derives, on the one hand, from studies of the stimulus prefix effect (e.g., Neisser, Hoenig, \& Goldstein, 1969) and, on the other, from studies of the stimulus suffix effect (e.g., Morton, Crowder, \& Prussin, 1971). In the cases of both the stimulus prefix and stimulus suffix, a redundant element, such as the word "zero," is included in the memory stimulus, a series of seven or eight digits, but is not required in recall. In both cases, the redundant element is presented in rhythm with the memory series and in the same modality. The two cases lie operationally on a continuum identified by the location of the redundant element in the presentation of the memory series; the redundant zero precedes the memory series in the one case and follows it in the other. While the inhibitory effect on overall recall may be about the same in these two cases, the inhibitory effect of the stimulus prefix is distributed equally over all items, whereas the effect of the stimulus suffix is specific to the terminal items of the memory series.

Different theoretical explanations have been advanced for these two effects. A stimulus prefix is presumed to increase the length of the memory stimulus and, therefore, the overall load on memory, because the prefix cannot be dissociated from the memory series proper (Neisser, Hoenig, \& Goldstein, 1969). However, the effect of the stimulus suffix is thought to be mediated by a precategorical acoustic storage (PAS) mechanism; a stimulus suffix increases the retention interval and, more importantly, is presumed to degrade the echoic traces of the terminal items of the memory series (Morton, Crowder, \& Prussin, 1971).

Because considerable attention has been given the stimulus suffix (e.g., Crowder, 1971a, b, 1975; Crowder \& Morton, 1969; Morton, Crowder, \& Prussin, 1971),

This research was supported by a grant from the Faculty Research Committee, Miami University. Requests for reprints should be sent to John C. Jahnke, Department of Psychology, Miami University, Oxford, Ohio 45056. it is perhaps surprising that so little is known about the nature of the stimulus prefix. Dallett (1964), who apparently was the first to demonstrate the stimulus prefix effect, showed that its effect on overall recall was statistically indistinguishable from that obtained by adding an extra nonredundant element to the memory series. In a second experiment (Dallett, 1965), the location of a single redundant digit, zero, was systematically varied in the memory series. Even though only the seven nonredundant digits were to be recalled, performance for conditions containing the redundant element was similar to that of the eight-digit control series rather than the seven-digit control series. When the zero was located in the final serial position and, therefore, was a suffix to the memory series, performance was very poor; the suffix effect was not restricted to the terminal items of the memory series in this experiment, for reasons that are unknown.

Neisser et al. (1969) showed that, while a single redundant zero used as a stimulus prefix had the expected inhibitory effect on recall, a sequence of three redundant prefix zeros actually improved recall of the memory series, as did a condition in which the redundant element was spoken in a male voice and the memory series in a female voice. The use of three zeros rather than one, and the presentation of the memory series and the stimulus prefix in different voices rather than one, were presumably to have made the redundant prefix element(s) readily discriminable and, therefore, dissociable from the memory series proper. In this way, memory load was smaller, and recall of the memory series was enhanced.

It was the purpose of the present study to gather more information on the effect of amount and location of stimulus redundancy on immediate recall. The present study examines, first, the effects of either one or two redundant elements used as a stimulus prefix; second, these effects are contrasted with those obtained when one or two redundant elements are used as suffixes and when a single-element prefix is combined with a 
Table 1

Proportion Correct Recall Overall for Each Experimental Condition

\begin{tabular}{cccc}
\hline Condition & $\begin{array}{c}\text { Proportion } \\
\text { Correct }\end{array}$ & Condition & $\begin{array}{c}\text { Proportion } \\
\text { Correct }\end{array}$ \\
\hline $07: 7$ & .80 & $007: 7$ & .78 \\
$07: 07$ & .65 & $007: 007$ & .64 \\
$70: 7$ & .70 & $700: 7$ & .66 \\
$70: 70$ & .69 & $700: 700$ & .63 \\
& & $070: 7$ & .65 \\
$7: 7$ & .83 & $070: 070$ & .56 \\
\hline
\end{tabular}

single element suffix. Third, new control conditions are included; these conditions require redundancy both in the stimulus during presentation and during recall. Thus, evidence is provided on the role of overt emission (vocalization) of the redundant element on the magnitude of both the prefix and suffix effects.

\section{METHOD}

\section{Subjects}

A total of 44 Miami University students, 22 male and 22 female, chose to participate in this experiment as an option of a service requirement for the introductory psychology course. None had previously served in any experiment on shortterm memory.

\section{Design and Materials}

There were 11 experimental conditions, each represented by a block of nine strings of digits. Each string was composed of seven digits that were supplemented by zero, one, or two occurrences of the redundant digit, zero. The seven digits were different permutations of the decimal digits, one through nine. The first two strings of each block were considered practice and the data from these were discarded. Each subject served in each condition; there were 11 different orders of presentation of the 11 conditions, arranged so that each condition occurred equally often at each stage of practice.

A convenient way to view the conditions of the present study is as five pairs of conditions, one of each pair an experimental condition and the other one a control, and an 11th (traditional) control condition. The five experimental conditions of the five

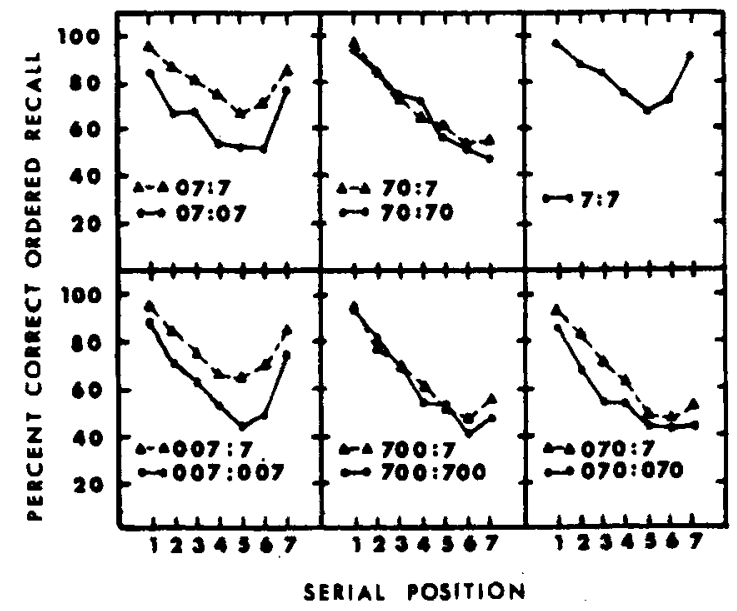

Figure 1. Serial position curves for the 11 experimental conditions. pairs examine the effect of location and amount of redundancy in the stimulus. The five paired control conditions require the stimulus redundancy also to be maintained overtly in recall. The five pairs of conditions follow: (a) In the stimulus prefix $(07: 7)$ condition, a single redundant element preceded the basic string of seven digits in presentation but was not to be given in recall. The paired control for this condition was the 07:07 condition, in which the redundant zero was given in presentation and was required in recall before any of the digits of the basic string were given. (b) The stimulus biprefix (007:7) condition was like the stimulus prefix condition, except that two redundant zeros preceded the presentation of the basic string of seven digits. The paired control for this condition was the 007:007 condition, for which the two redundant digits were also required in recall. (c) In the stimulus suffix $(70: 7)$ condition, the redundant digit followed the basic string of seven digits in presentation and was not to be given in recall. Its paired control was the $70: 70$ condition, in which the redundant element was also given in recall but after the recall of the basic string. (d) In the stimulus bisuffix (700:7) condition, the two redundant zeros followed the basic string, but only the seven digits of the basic string were to be given in recall. Its paired control was the 700:700 condition, in which the redundant elements were included in presentation and were to be given in recall subsequent to the recall of the basic string. (e) In the combined stimulus prefix-suffix $(070: 7)$ condition, a redundant zero preceded and followed the basic string, but only the basic string was to be given in recall. The paired control was the 070:070 condition, in which the redundant digits were to be included in recall in positions corresponding to their positions in presentation. The 11 th condition was the $7: 7$ condition, a traditional control condition that included no redundant elements either in presentation or recall.

\section{Procedure}

The strings were presented auditorially. The digits of the strings were tape recorded in male voice, speaking at a $2 \mathrm{sec}$ rate. ${ }^{1}$ A brief $440-\mathrm{Hz}$ tone was presented before each string, as a ready signal, and after, as a signal to begin recall. The intertrial (interstring) interval, during which recall was given orally, was $10 \mathrm{sec}$. The subjects were asked to recall items in their order of presentation and to say "blank" each time they could not recall a given item. Instructions appropriate to each of the 11 conditions were given prior to the block of trials representing that condition. Subjects served individually in an experimental session that was about 45 min long.

\section{RESULTS}

For all analyses reported here, an item had to be recalled in its proper serial position in order to be scored as correct. There were no errors in the recall of the redundant elements, except in the $70: 70$, $700: 700$, and $070: 070$ conditions, when some subjects ended some of their recalls with the required number of zeros, but these elements were not in the proper serial positions. A total of 15 subjects made a total of 49 such errors (out of 924 possible), and 28 of these errors were accounted for by just three subjects. Table 1 and Figure 1, respectively, show the overall proportion correct recall and the serial position functions for the basic seven-digit series of nonredundant elements. Table 1 and Figure 1 show that performance was better, at least marginally so, for the experimental member of the five pairs of conditions than for the correspond- 
ing control member, and that performance was best of all in the $7: 7$ control condition that contained no redundant elements either in presentation or recall.

The comparison of performance in the $7: 7$ control condition with that in the 07:7 and 007:7 conditions suggests that there is a small stimulus prefix effect that increases slightly with the presence of a second prefix element. The comparison of performance in the $7: 7$ control with that in the $70: 7$ and $700: 7$ conditions indicates a pronounced stimulus suffix effect that, relative to the performance of its paired control, also increases somewhat with the addition of a second suffix element. Performance in the 070:7 (combined prefixsuffix) condition appears to be about equivalent to that in the suffix conditions. The serial position curves for the 7:7 condition and the 07:7 and 007:7 conditions and their corresponding controls displayed in Figure 1 all show the prominent bowing associated with ordered recall. The serial position curves for the remaining conditions, all of which involve a stimulus suffix, show little bowing and the expected poor performance on the terminal item(s) of the string.

A related-measures analysis of variance that included serial position as a variable was performed on the data on which Figure 1 is based. This analysis showed that main effects of conditions and serial position were significant at the .01 level (the rejection region used for this and all subsequent analyses), $F(10,430)=28.48$, $\mathrm{MS}_{\mathrm{e}}=3.68$, and $\mathrm{F}(6,258)=134.67, \mathrm{MS}_{\mathrm{e}}=3.43$; the interaction of these two variables was also significant, $\mathrm{F}(60,2520)=10.27, \mathrm{MS}_{\mathrm{e}}=97$. A Newman-Keuls analysis showed that performance in the stimulus prefix conditions did not differ significantly from that in the $7: 7$ control, whereas each of the two stimulus suffix conditions and the combined prefix-suffix condition did. This analysis also indicated that the $70: 7$ and 700:7 suffix conditions and the 070:7 condition, which did not differ in terms of overall performance, differed significantly from the 07:7 and 007:7 prefix conditions, which also did not differ between themselves.

It was also found that performance was significantly better overall for the stimulus prefix and biprefix conditions than their corresponding controls, as was also true for the comparison involving the combined prefix-suffix condition and its control. Neither the difference between the $70: 7$ suffix condition and its paired control nor the $700: 7$ bisuffix condition and its paired control was significant overall. However, performance on the terminal item of the $70: 7$ condition differed from that of its control; performance on each of the last two items of the $700: 7$ condition differed from that of its control.

The serial position data of Figure 1 show that the location of the redundant elements had a considerable effect on performance, a fact reflected in the significant interaction mentioned above. As noted earlier, each condition involving a stimulus suffix was associated with poor performance on (at least) the terminal item of the memory series. On the other hand, the stimulus prefix conditions seem not to have had any positionspecific effect, and the Newman-Keuls analysis showed that any contrast involving either of the two prefix conditions with any stimulus suffix condition (including the combined prefix-suffix condition) was statistically significant at both Serial Positions 6 and 7 .

A supplement to the serial position analyses presented here is one that corrects for the different absolute performance levels associated with the various experimental conditions. Such a transformation of the data of Figure 1 was accomplished by determining, for each condition separately, the frequency of correct recall at each serial position relative to the frequency of correct recall overall for that condition. While these data are not presented here, they showed that the serial position functions of the prefix, biprefix, and the $7: 7$ control conditions overlapped considerably, as would be expected. The functions for the suffix, bisuffix, and the combined prefix-suffix conditions, on the other hand, showed both enhanced primacy and the expected degraded recency, relative to the $7: 7$ control. Perhaps of special interest was the result that performance at Serial Position 7 was slightly higher for the bisuffix condition than for the suffix and the combined prefixsuffix conditions, for which performance levels at this and other serial positions were quite comparable.

\section{DISCUSSION}

Overall performance was best in Condition $7: 7$, which contained no redundant elements in either the stimulus or recall. While the inclusion of a redundant element in the stimulus degraded recall overall, this effect was not significant for either the 07:7 or 007:7 prefix conditions. Since the inhibitory effect of a stimulus prefix is ordinarily quite pronounced, it is suggested tentatively that exposure to the variety of conditions of redundancy in the present experiment enhanced the subject's ability to organize perceptually the stimulus and, in this way, lessened the magnitude of the prefix effect.

Major emphasis, however, should be given to two groups of findings. The first is that overall performance in the combined prefix-suffix $(070: 7)$ condition was equivalent to that in the two suffix $(70: 7$ and $700: 7)$ conditions and was poorer in these three conditions than in the two prefix conditions. If the effect of a suffix is to erase items from PAS, a single suffix seems to suffice as well as two; performance in these three conditions differed only at Serial Position 5, where 70:7 performance exceeded that in each of the two remaining conditions. The second is that the comparison of the $07: 7,007: 7$, and $070: 7$ conditions, all of which include a stimulus prefix, with their correspond- 
ing controls shows that requiring the redundant element(s) to be given in recall significantly worsened recall overall. Even if the stimulus prefix is encoded with the memory series during presentation, it apparently induces little or no interference in recall, relative to a condition in which the prefix is required in recall. In the latter case, output interference associated with the overt recall of the prefix is implicated as a factor important to the inhibitory effect. In contrast, the comparison of the $70: 7$ and $700: 7$ suffix conditions with their corresponding controls shows no inhibitory effect on overall recall, although performance was selectively poorer on the terminal item in Condition 70:70 than in 70:7 and on the last two items in Condition 700:700 than in 700:7. To this degree, output interference also seems important to the difference between the suffix experimental and control conditions. That performance was poorer in the suffix control conditions than in the experimental is particularly interesting, since it is consistent with the notion that the stimulus suffix requires cognitive processing capacity in order to be maintained for recall, and that this processing interferes with recall of (at least) the terminal nonredundant item.

The major result of the present experiment is that stimulus prefixes and stimulus suffixes affect recall differentially. The effect of the stimulus suffix was the greater of the two and was, as expected, serial position specific. Previously it had been supposed that prefix and suffix effects are mediated by two different mechanisms. It is proposed here, however, that both effects may be accounted for by presuming that the subject usually is unable to dissociate fully the redundant element from the nonredundant elements. Because a prefix precedes the memory series, it neither delays recall nor degrades echoic traces of the memory series: as does a suffix; hence, the effect of the stimulus suffix is different in form from, and may be greater than, that of a stimulus prefix.

\section{REFERENCES}

Crowder, R. G. The sound of vowels and consonants in immediate memory. Joumal of Verbal Leaming and Verbal Behavior, 1971, 10, 587-596. (a)

Crowder. R. G. Waiting for the stimulus suffix: Decay, delay, rhythm, and readout in immediate memory. Quarterly Journal of Experimental Psychology. 1971, 23, 324-340. (b)

CROWDer, R. G. Inferential problems in echoic memory. In P. M. A. Rabbit \& S. Dornic (Eds.), Attention and performance V. New York: Academic Press, 1975. Pp. 218-229.

Crowder. R. G.. \& Morton, J. Precategorical acoustic storage (PAS). Perception \& Psychophysics, 1969, 5, 365-373.

DalletT, K. M. Effects of a redundant prefix on immediate recall. Journal of Experimental Psychology, 1964, 67, 296-298.

DalletT, K. M. "Primary memory": The effects of redundancy upon digit repetition. Psychonomic Science, 1965, 3, 237-238.

Morton, J., Crowder, R. G., \& Prussin, H. A. Experiments with the stimulus suffix. Journal of Experimental Psychology Monograph, 1971. 91, 169-190.

Neisser, U., Hoenig, Y., \& Goldstein, E. Perceptual organization and the prefix effect. Journal of Verbal Learning and Verbal Behavior. 1969, 8, 424-429. (Received for publication August 27, 1975:
revision accepted October 21, 1975.)

\section{NOTE}

1. A perceptive reviewer has drawn attention to a possible limitation of the procedure: It is conceivable that the reading of the digits is influenced by the same variables that influence memory for the digits. Therefore, the subject's recall could reflect more the experimenter's attentional processes than the subject's attentional and memorial ones. Of course, several solutions to such a problem immediately come to mind. 